\title{
Perfil dos pacientes com gangrena de Fournier utilizando a oxigenoterapia hiperbárica como tratamento adjuvante
}

\author{
Profile of Fournier gangrene patients using hyperbaric oxygen therapy as an \\ adjuvant treatment
}

Katia Fumika Tikami ${ }^{1}$ (D), José Cláudio Simão² (D), Larissa Camargo Passerotti ${ }^{\text {(D), }}$ Adriana Sierra Assencio Almeida Barbosa ${ }^{4}$ (D)

\begin{abstract}
RESUMO
Objetivo: Avaliar os resultados obtidos da oxigenoterapia hiperbárica (OHB) como tratamento adjuvante na Gangrena de Fournier (GF). Casuística e Método: Estudo transversal, retrospectivo e descritivo baseado na análise de prontuários físicos de pacientes portadores de GF submetidos a oxigenoterapia hiperbárica, através da câmara hiperbárica no período de fevereiro de 2008 a maio de 2018, atendidos em uma clínica de oxigenoterapia hiperbárica na cidade de Bauru, SP. Os dados coletados foram: idade, sexo, etiologia, comorbidades, localização e extensão da lesão, número total de sessão e mortalidade. Resultado: Trinta e oito pacientes foram analisados, sendo $76 \%$ do sexo masculino, com média de idade de 55,6 anos. Entre os pacientes, $17(44,7 \%)$ obtiveram alta médica, 18 $(47,4 \%)$ interromperam o tratamento antes do término e ocorreram três $(8,21 \%)$ óbitos. A principal etiologia foi o abcesso e os principais comorbidades predisponentes foram a diabetes mellitus e a hipertensão arterial. A localização e extensão com áreas mais afetadas foram na região escrotal, seguida pela região perineal. Conclusão: Os resultados deste estudo demostraram que o efeito da OHB como tratamento adjuvante oferece vantagem no tratamento da GF, resultando em considerável alta médica dos pacientes e baixa mortalidade.
\end{abstract}

Palavras-Chave: Gangrena de Fournier; Oxigenação Hiperbárica; Terapia Combinada.

\begin{abstract}
Objective: Evaluating the results obtained from hyperbaric oxygen therapy (HBOT) as an adjuvant treatment in Fournier's Gangrene (GF). Casuistic and Method: Cross-sectional, retrospective, and descriptive study based on the analysis of physical records of patients with GF submitted to hyperbaric oxygen therapy through the hyperbaric chamber from February 2008 to May 2018, assisted at the hyperbaric oxygen therapy clinic, in Bauru, SP. The data collected were age, gender, etiology, comorbidities, lesion location and extension, total session number, and mortality. Result: It totaled 38 patients, $76 \%$ male, with a mean age of 55.6 years. Among the patients, 17 (44.7\%) were discharged, $18(47.4 \%)$ discontinued treatment before termination, and three $(8.21 \%)$ deaths occurred. The main etiology was an abscess and the main predisposing comorbidities were diabetes mellitus and hypertension. The location and extension lesion with the most affected areas were in the scrotal region, followed by the perineal region. Conclusion: The results of this study demonstrated that the effect of HBOT as an adjuvant treatment has an advantage in the treatment of GF resulting in considerable patient medical discharge and low mortality.
\end{abstract}

Keywords: Fournier Gangrene; Hyperbaric Oxygenation; Combined Modality Therapy.

1. Tecnóloga em Sistemas Biomédicos pela Faculdade de Tecnologia de Bauru -FATEC Bauru (SP), Brasil.

2. Doutor em Doenças Tropicais pela Universidade Estadual Paulista Júlio de Mesquita Filho (UNESP), Botucatu (SP), Brasil.

3. Médica Hiperbarista e Nefrologista pediátrica responsável Técnica junto a empresa Oxibarimed Servicos de Oxigenoterapia Hiperbárica Ltda.

4. Docente na Faculdade de Tecnologia de Bauru- FATEC Bauru (SP), Brasil.

$\bowtie$ Adriana Sierra Assencio Almeida Barbosa. Rua Manoel Bento Cruz, 3-30, Centro. CEP: 17015-171. Bauru (SP), Brasil. drisierra@hotmail.com | Recebido em: 01/10/2019 | Aprovado em: 24/01/2020 


\section{INTRODUÇÃO}

Síndrome de Fournier ou Gangrena de Fournier (GF) é uma fasceíte necrotizante de origem polimicrobiana causada por micro-organismos aeróbicos e anaeróbicos. Acomete principalmente as regiões genital, perianal e perineal ${ }^{1,2}$. Em alguns estudos, há relatos de um histórico nestes pacientes de traumatismo perineal, doença colorretal, infecção urinária, escara de decúbito, além de causa desconhecida ${ }^{1,2}$.

Frequentemente ela se inicia com dor, febre, eritema, edema e necrose escrotal ou na região perianal e perineal. As manifestações cutâneas podem também não se manifestar, uma vez que a infecção pode-se alastrar ao longo dos planos fasciais profundos ${ }^{3,4}$.

Estudos demonstram que na GF encontram-se uma associação de bactérias aeróbicas e anaeróbicas normalmente não patogênicas, mas mortais quando em locais não habituais. Essas bactérias podem agir de forma sinérgica, contribuindo, com isso, na rápida evolução e aumento da área disseminada, agravando o quadro da doença ${ }^{5,6}$.

A infecção se caracteriza por uma endarterite obliterante que pode evoluir em uma isquemia e trombose vascular cutânea e subcutânea, que resulta em necrose da pele e tecido celular subcutâneo adjacente. Se este processo não for rapidamente diagnosticado e tratado, pode-se espalhar à parte abdominal anterior, à região dorsal, aos membros superiores e ao retroperitônio, podendo evoluir à sepse, falência de múltiplos órgãos e à morte ${ }^{7,8}$.

A GF está associada a várias comorbidades como diabetes mellitus, alcoolismo, tabagismo, hipertensão arterial, obesidade, doenças imunossupressoras como HIV, radioterapia, quimioterapia, leucemia, entre outras ${ }^{9,10}$.

O tratamento da GF exige uma equipe multidisciplinar, variando de acordo com as necessidades de cada caso, com a necessidade de desbridamento cirúrgico de emergência com ou sem derivação fecal ou urinária, antibioticoterapia de largo espectro e terapia adjuvantes como a oxigenoterapia hiperbárica $(\mathrm{OHB})^{11}$.

Medidas adjuvantes como a (OHB) são utilizadas para prevenir a extensão da necrose, reduzir sinais sistêmicos da infecção e melhorar a recuperação do tecido. Essa terapia acelera a cicatrização após cirurgia e, por ter efeito antibacteriano, reduz a necessidade de sucessivos desbridamentos ${ }^{12,13}$.

A OHB consiste na exposição do paciente a inalação de oxigênio puro a $100 \%$, em temperatura ambiente, sob pressão superior (geralmente duas a três vezes) a pressão atmosférica ao nível do mar. Este aumento de pressão resulta em aumento da pressão arterial e tecidual de oxigênio (2000 mmHg e $400 \mathrm{mmHg}$, respectivamente), o que está na base da maioria dos efeitos fisiológicos e terapêuticos ${ }^{12,13}$.

O mecanismo de ação da OHB proporciona efeitos bioquímicos e celulares, resultando na cicatrização de feridas e a neovascularização, além de possuir ação antimicrobiana. A OHB estimula a ativação dos leucócitos em hipóxia que apresentam dificuldade na fagocitose de micro-organismo e a síntese de colágeno pelos fibroblastos aumenta com a maior disponibilidade de oxigênio. O aumento da oxigenação tecidual restaura a angiogênese capilar, aumentando a proliferação tecidual e a formação do tecido de granulação ${ }^{14,15}$. Nos pacientes usuários da OHB ocorre um aumento na pressão parcial de oxigênio no sangue arterial com elevação do oxigênio entre os capilares e os tecidos, aumentando a oxigenação celular e quebrando o ciclo vicioso da isquemia, levando a vasoconstrição e consequente redução de edemas e de pressões compartimentais ${ }^{14,15}$.

A OHB é uma medida auxiliadora no tratamento clássico da GF, diminuindo a mortalidade e a quantidade de tecido a ser removido cirurgicamente, pois, ao remover os exsudatos, promove a cobertura da ferida por tecido de granulação, estimulando a angiogênese e reduzindo a contaminação bacteriana na $\mathrm{GF}^{16}$.

Este equipamento é seguro e eficaz na aplicação de diversas patologias. Assim, o presente estudo tem como objetivo avaliar os resultados obtidos da OHB como tratamento adjuvante na GF.

\section{CASUISTICA E MÉTODO}

Trata-se de estudo transversal, retrospectivo e descritivo baseado na análise de prontuários físicos de pacientes portadores de GF submetidos 
a oxigenoterapia hiperbárica, através da câmara hiperbárica (modelo Multiplace A-240, marca Seaway Diver, Porto Alegre, Brasil), no período de fevereiro de 2008 a maio de 2018, atendidos em uma clínica de oxigenoterapia hiperbárica na cidade de Bauru, SP.

Os pacientes foram selecionados de acordo com os critérios de inclusão: pacientes com diagnóstico de GF e maiores de 18 anos de idade.

Os dados dos prontuários físicos foram registrados em ficha de coleta de dados com as informações de idade, sexo, etiologia, comorbidades, localização e extensão da lesão, número total de sessão e mortalidade.

Este estudo foi avaliado e aprovado pelo Comitê de Ética em Pesquisa do Instituto Lauro de Souza Lima, parecer número 2.657.698/2017.

Os dados foram analisados descritivamente utilizando o programa para microcomputador Statistical Package for Social Science (SPSS), versão 17.0 para Windows.

\section{RESULTADOS}

Através da análise dos prontuários, foram avaliados 38 pacientes com o diagnóstico de gangrena de Fournier, num total de 29 pacientes do sexo masculino $(76 \%)$ e nove do sexo feminino (24\%). A idade média foi de 55,6 anos, variando de 29 a 82 anos.

Entre os pacientes, 17 (44,7\%) obtiveram alta médica, $18(47,4 \%)$ interromperam o tratamento antes do término e ocorreram três $(8,21 \%)$ óbitos. O período de tratamento de $71 \%$ dos casos foi de 2,6 meses, com variação de 1 a 5 meses. A média do número total de sessões foi de 29, variando de 3 a 55 sessões.

A Tabela 1 apresenta a etiologia mais comum sendo o abscesso, observado em dez $(26,3 \%)$ pacientes. Na etiologia denominada *Outros, estão inclusos os fatores como: alteração urológica, herpes Zoster, infecção indeterminada, hérnia, cisto pilonidal e fístula, os quais apresentam um único caso cada, representando individualmente $2,63 \%$.

A Tabela 2 exibe as principais comorbidades nos pacientes com GF que foram de 60 fatores associados, pois alguns pacientes apresentam mais de um fator simultaneamente. A principal comorbidade foi hipertensão arterial sistêmica com 17 (28,3\%) casos, seguida por diabetes mellitus com 15 (25\%) casos. Não houve um fator comum entre os pacientes que foram a óbito. Em *Outros estão incluídas as comorbidades que apresentaram um único caso, como a paraplegia, hepatopatia, constipação, orquiectomia, acidente vascular periférico, trombose venosa, dislipidemia, colecistectomia, cardiopatia e gota, cada uma representando um percentual individual de $1,68 \%$.

\section{Tabela 1}

Etiologia da gangrena de Fournier

\begin{tabular}{lcc}
\hline Etiologia & Quantidade & Porcentagem (\%) \\
\hline Abscesso & 10 & 26,3 \\
Trauma & 4 & 10,5 \\
Lesão, ferimento & 4 & 10,5 \\
Escara & 3 & 7,9 \\
Furúnculo & 3 & 7,9 \\
Edema & 3 & 7,9 \\
Causa desconhecida & 3 & 7,9 \\
Hemorroida & 2 & 5,3 \\
$*$ Outros & 6 & 15,8 \\
\hline
\end{tabular}

\section{Tabela 2}

Principais comorbidades nos pacientes com gangrena de Fournier

\begin{tabular}{lcc}
\hline Comorbidades & Quantidade & Porcentagem (\%) \\
\hline Hipertensão arterial & 17 & 28,3 \\
sistêmica & 15 & 25,0 \\
Diabetes & 5 & 8,3 \\
Sem predisposição & 4 & 6,7 \\
Obesidade & 3 & 5,0 \\
Quimioterapia & 2 & 3,3 \\
Etilismo & 2 & 3,3 \\
Lesão & 2 & 3,3 \\
Tabagismo & 10 & 16,8 \\
*Outros & & \\
\hline
\end{tabular}

Quanto à localização e extensão da lesão, observou-se que no início do tratamento alguns pacientes apresentaram dois ou três lugares distintos simultaneamente, totalizando 68 regiões. O local mais atingido foi a região escrotal com 17 (25\%) casos e a região perianal/perineal com 16 $(23,5 \%)$ casos, como apresenta a Tabela 3. 
Tabela 3

Localização e extensão da lesão

\begin{tabular}{lcc}
\hline Local & Quantidade & Porcentagem (\%) \\
\hline Escroto & 17 & 25,0 \\
Perineal & 16 & 23,5 \\
Inguinal & 11 & 16,2 \\
Pênis & 8 & 11,8 \\
Glúteo & 4 & 5,9 \\
Supra púbica & 4 & 5,9 \\
Sacral & 3 & 4,4 \\
Vagina & 3 & 4,4 \\
Abdômen & 2 & 2,9 \\
\hline
\end{tabular}

\section{DISCUSSÃO}

A gangrena de Fournier é um tipo de fasceíte necrotizante que pode espalhar rapidamente para outras regiões e membros do corpo, podendo evoluir rapidamente para o óbito ${ }^{1}$. O tratamento da GF se baseia pelo desbridamento cirúrgico, antibioticoterapia de amplo espectro e terapia adjuvantes como a oxigenoterapia hiperbárica (OHB) ${ }^{2}$.

A OHB é utilizada com segurança e eficácia para uma ampla gama de patologias, tendo a sua eficácia no tratamento da GF demonstrada em vários estudos, uma vez que remove exsudatos, promove a cobertura da ferida, estimula a angiogênese e reduz a contaminação bacteriana ${ }^{3,4,9,10}$.

Analisando os resultados, observa-se que o perfil do paciente com GF do presente estudo foi do sexo masculino, com média de idade de 55,6 anos, concordando com a literatura ${ }^{2,5,10,12}$.

Pesquisas realizadas com pacientes com GF relatam que a proporção de afetados são de dez pacientes do sexo masculino para um paciente do sexo feminino $2,5,7,10,11$. A proporção encontrada no presente estudo foi sete pacientes do sexo masculino para três do sexo feminino. A proporção do sexo feminino resulta em um perfil da mulher moderna com jornada dupla de trabalho, entre atribuições profissionais e atividade doméstica, dispondo-a de menos tempo para o cuidado com sua saúde.

Estudos realizados relatam que a etiologia da GF são abscesso, traumatismo, infecções, escara e causa desconhecida ${ }^{1,3,6,9,15}$. No presente estudo a etiologia mais comum foi o abscesso em $26,3 \%$, sendo que a localização e a extensão da lesão mais afetada foram escroto e perineal, totalizando $48,5 \%$ dos casos.

Algumas comorbidades são predisponentes para o desenvolvimento da GF. O diabetes mellitus e hipertensão arterial são encontrados na maioria dos indivíduos com a gangrena, de acordo com trabalhos já publicados $2,4,8,12,16$. Outros fatores também estão associados à GF como: alcoolismo, tabagismo, obesidade, quimioterapia, entre outros. Os dados encontrados no presente estudo corroboram com a literatura.

A taxa de mortalidade encontrada no presente estudo foi de $8,21 \%$. Os números de mortalidade são variáveis na literatura, apesar das melhorias no diagnóstico e tratamento. Mehl et al. (2010)11 relataram que os pacientes submetidos à $\mathrm{OHB}$ apresentaram, proporcionalmente, menor índice de mortalidade de $11,5 \%$ comparado com os que não receberam a taxa foi de $37,5 \%$. 0 artigo de revisão publicado por Sroczyński et al. (2013) 4 relata que em pacientes que receberam a OHB no tratamento de GF, a taxa de mortalidade foi menor que $20 \%$, comparado a não utilização da terapia com OHB, a variação foi de 18 a $50 \%$. Estudo realizado por Li et al. (2015) $)^{15}$ comparando a taxa de mortalidade encontrou diferenças entre os grupos de pacientes com GF utilizando a OHB de $12,5 \%$ e para o grupo tratado sem a terapia com OHB, de 33,3\%.

Estudo conduzido por Devaney et al. $(2015)^{12}$ relatou uma taxa de mortalidade de $14,4 \%$ na pesquisa com 341 pacientes com GF tratados com OHB. Segundo Anheuser et al. (2018) ${ }^{14}$, em estudo realizado com 17 pacientes tratados com $\mathrm{OHB}$, a taxa de mortalidade foi de $0,0 \%$.

Os dados do presente estudo demonstram a eficácia da terapia adjuvante com OHB devido à melhora da patologia através da alta médica, corroborando com pesquisas publicadas por diversos autores $7,8,9,12,13,14,15$.

Os resultados encontrados no presente estudo quanto à indicação da OHB para o número de sessões foram de uma média de 29 , demonstrando semelhanças com pesquisas realizadas ${ }^{8,13,15}$.

A quantidade de desistência encontrada no presente estudo foi de $47,4 \%$, levantando muitos questionamentos sobre sua ocorrência pois, em pacientes que obtiveram alta, o tratamento durou de 1 a 5 meses, nos casos de maior extensão. Muitos 
pacientes deste estudo são moradores de outras cidades, demandando uma dedicação e comprometimento maior. As lesões em muitos pacientes são extensas e o seu estado físico e psicológico necessita de um cuidado especial na locomoção e curativos. Convênios médicos particulares, na maioria das vezes, impõem dificuldades para renovar a continuidade do tratamento quando há necessidade de mais sessões do que inicialmente indicado. Existe também a cultura psicossocial do próprio paciente se considerar curado e parar o tratamento.

Assim como qualquer outra modalidade, o tratamento adjuvante a OHB apresenta algumas limitações como: equipamento adequado, disponibilidade do paciente em aderir ao tratamento, disponibilidade de locais restritos, pouca divulgação do tratamento.

\section{CONCLUSÃO}

Os resultados deste estudo demostraram que o efeito da OHB como tratamento adjuvante oferece vantagem no tratamento da GF, resultando em considerável alta médica dos pacientes e baixa mortalidade.

Contudo, a OHB está se tornando uma opção de tratamento cada vez mais utilizada, pois promove o reparo e a cicatrização de tecidos, facilitando a angiogênese e o crescimento de fibroblastos, estimulando a atividade anti-inflamatória.

Portanto, através dos dados encontrados, sugere-se que é necessária uma vasta divulgação da eficiência do tratamento com a OHB, podendo ampliar a quantidade de equipamentos disponíveis para comunidade, resultando em uma opção de terapia segura e eficaz para uma extensa gama de patologias.

\section{REFERÊNCIAS}

1. Milanese G, Quaresima L, Dellabella M, Scalise A, Di Benedetto GM, Muzzonigro G, et al. A conservative approach to perineal Fournier's gangrene. Archivio Italiano di Urol Androl. 2015;87(1):28-32. doi: 10.4081/aiua.2015.1.28

2. Cardoso JB, Féres O. Gangrena de Fournier. Medicina (Ribeirão Preto). 2007;40(4): 493-9. doi: 10.11606/ issn.2176-7262.v40i4p493-499
3. Dornelas MT, Correa MPD, Barra FML, Corrêa LD, Silva C, Dornelas GV, et al. Síndrome de Fournier: 10 anos de avaliação. Rev Bras Cir Plast. 2012;27(4):600-4. doi: $10.1590 / S 1983-51752012000400022$

4. Sroczyński M, Sebastian M, Rudinicki J, Sebastian S, Agrawal AK. A complex approach to the treatment of Fournier's gangrene. Adv Clin Exp Med. 2013;22(1):407-10.

5. Santos DR, Roman ULT, Westphalen AP, Lovison K, Spencer NFAC. Profile of patients with Fournier's gangrene and their clinical evolution. Rev Col Bras Cir. 2018;45(1):e1430. doi: 10.1590/0100-6991e-20181430

6. Benjelloun EB, Souiki T, Yakla N, Ousaddean A, Mazaz K, Louchi $A$, et al. Fournier's gangrene: our experience with 50 patients and analysis of factors affecting mortality. World J Emerg Surg. 2013;8(1):13. doi: 10.1186/17497922-8-13

7. Rosa I, Guerreiro F. Hyperbaric oxygen therapy for the treatment of Fournier's gangrene: a review of 34 cases. Acta Med Port. 2015; 28:619-23. doi: 10.20344/amp-6300

8. Hung MC, Chou CL, Cheng LC, Ho CH, Niu KC, Chen HL, et al. The role of hyperbaric oxygen therapy in treating extensive Fournier's gangrene. Urol Sci. 2016;27:148-153. doi: $10.1016 /$ j.urols.2015.06.294

9. Pastore AL, Palleschi G, Ripoli A, Silvestri L, Leto A, Autieri D, et al. A multistep approach to manage Fournier's gangrene in a patient with unknown type II diabetes: surgery, hyperbaric oxygen, and vacuum-assisted closure therapy: a case report. J Med Case Rep. 2013;7:1. doi: $10.1186 / 1752-1947-7-1$

10. Ferretti M, Saji AA, Phillips J. Fournier's Gangrene: A Review and Outcome Comparison from 2009 to 2016. Adv Wound Care. 2017;6(9):289-95. doi: 10.1089/wound.2017.0730

11. Mehl AA, Nogueira Filho DC, Mantovani LM, Grippa MM, Berger $R$, Krauss $D$, et al. Manejo da gangrena de Fournier: experiência de um Hospital Universitário de Curitiba. Rev Col Bras Cir. 2010;37(6). doi: 10.1590/ S0100-69912010000600010

12. Devaney B, Frawley G, Frawley L, Pilcher DV. Necrotizing soft tissue infections: the effect of hyperbaric oxygen on mortality. Anaesth Intensive Care. 2015;43(6):685-92. doi: $10.1177 / 0310057 \times 1504300604$

13. Gandhi J, Seyam O, Smith NL, Joshi G, Vatsia S, Khan SA. Clinical utility of hyperbaric oxygen therapy in genitourinary medicine. Med Gas Res. 2018;8(1):29-33. doi: 10.4103/2045-9912.229601

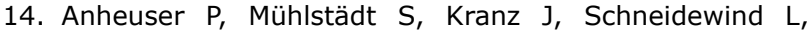
Steffens J, Fornara P. Significance of Hyperbaric Oxygenation in the Treatment of Fournier's Gangrene: A comparative Study. Urol Int. 2018;101(4):467-71. doi: $10.1159 / 000493898$

15. Li C, Zhou X, Liu LF, Qi F, Chen JB, Zu XB. Hyperbaric oxygen therapy as an adjuvant therapy for comprehensive treatment of Fournier's gangrene. Urol Int. 2015;94:453-458. doi: 10.1159/000366137

16. Sorensen MD, Krieger JN: Fournier's Gangrene: epidemiology and outcomes in the general US population. Urol Int. 2016;97:249-59. doi: 10.1159/000445695 